\title{
Estimates for the multiple singular integrals via extrapolation
}

\author{
Meng Qu
}

Correspondence: qumeng@mail. ahnu.edu.cn

School of Mathematical and

Computer Sciences, Anhui Normal University, Wuhu, 241002, People's

Republic of China

\section{Abstract}

This paper is devoted to the study on the $L^{p}$ estimates for the multiple singular integrals with rough kernels on product spaces $\mathbb{R}^{n} \times \mathbb{R}^{m}(n, m \geq 2)$. By means of extrapolation method and Fourier transform estimate, we prove that the multiple singular integral operators are bounded on $L^{p}\left(\mathbb{R}^{n} \times \mathbb{R}^{m}\right)$ for the kernel functions: $\Omega \in$ $L(\log L)^{2}\left(S^{n-1} \times S^{m-1}\right), h \in \widetilde{\Delta}_{\alpha}\left(\mathbb{R}^{+} \times \mathbb{R}^{+}\right)(\alpha \in(1,2])$. Furthermore, we prove that when $\Omega \in L(\log L)^{2}\left(S^{n-1} \times S^{m-1}\right)$ and $h$ satisfying a 'log' type condition defined on $\mathbb{R}^{+}$ $\times \mathbb{R}^{+}$, the multiple singular integral operators are bounded on $L^{2}\left(\mathbb{R}^{n} \times \mathbb{R}^{m}\right)$, which improves the well-known result.

$\operatorname{MR}(2010)$ Subject Classification

42B20, 42B25

Keywords: singular integral, Littlewood-Paley theory, rough kernel, extrapolation method

\section{Introduction}

Let $\mathbb{R}^{n}(n \geq 2)$ be $n$-dimensional Euclidian space and $S^{n-1}$ be the unit sphere in $\mathbb{R}^{n}$. Suppose that the function $\Omega \in L^{1}\left(S^{n-1}\right)$ satisfies the following cancelation condition

$$
\int_{S^{n-1}} \Omega(\theta) \mathrm{d} \sigma(\theta)=0,
$$

where $\mathrm{d} \sigma$ denotes the usual Lebesgue surface measure on the unit sphere $S^{n-1}$.

Let $L(\log L)^{\alpha}\left(S^{n-1}\right)$ denotes the functions $\Omega$ defined on $S^{n-1}$ satisfying the Zygmund condition: for $\alpha>0$,

$$
\int_{S^{n-1}}|\Omega(\theta)|(\log (2+|\Omega(\theta)|))^{\alpha} \mathrm{d} \sigma(\theta)<\infty .
$$

It is noted that for any $q>1$, we have the proper inclusion relations hold:

$$
\begin{gathered}
L^{q}\left(S^{n-1}\right) \subset L(\log L)^{\alpha}\left(S^{n-1}\right) \subset L^{1}\left(S^{n-1}\right), \\
L(\log L)^{\beta}\left(S^{n-1}\right) \subset L(\log L)^{\alpha}\left(S^{n-1}\right) \text { if } 0<\alpha<\beta .
\end{gathered}
$$

For $s \geq 1$, let $\Delta_{s}\left(\mathbb{R}^{+}\right)$denote the collection of measurable functions $h$ on $\mathbb{R}^{+}=\{t \in \mathbb{R}$ : $t>0\}$ satisfying

(C) 2011 Qu; licensee Springer. This is an Open Access article distributed under the terms of the Creative Commons Attribution License (http://creativecommons.org/licenses/by/2.0), which permits unrestricted use, distribution, and reproduction in any medium, provided the original work is properly cited. 


$$
\|h\|_{\Delta_{s}\left(\mathbb{R}^{+}\right)}=\sup _{j \in \mathbb{Z}}\left(\int_{2^{j}}^{2^{j+1}}|h(t)|^{s} d t / t\right)^{1 / s}<\infty,
$$

where $\mathbb{Z}$ denotes the set of integers. Also by usual modification, $\Delta_{\infty}\left(\mathbb{R}^{+}\right)=L^{\infty}\left(\mathbb{R}^{+}\right)$. We note that $\Delta_{s} \subset \Delta_{t}$ if $s>t$. We can always assume that $h \in \Delta_{1}$.

A singular integral operator is defined in the following form:

$$
S(f)(x)=p . v \cdot \int_{\mathbb{R}^{n}} f(x-y) K(y) \mathrm{d} y=\lim _{\varepsilon \rightarrow 0} \int_{|y|>\varepsilon} f(x-y) K(y) \mathrm{d} y,
$$

for an appropriate function $\mathrm{f}$ on $\mathbb{R}^{n}$, where $K(y)=|\mathrm{y}|^{-n} h(|y|) \Omega\left(y^{\prime}\right), y^{\prime}=|y|^{-1} y$.

It is well known that if $\Omega \in L \log L\left(S^{n-1}\right), h=1$, by the method of rotations, Calderón and Zygmund [1] proved that $S$ extends to a bounded operator on $L^{p}$ for all $p \in$ $(1, \infty)$. In [2], R. Fefferman first introduced the case of rough radial and proved that if $h \in \Delta_{\infty}\left(\mathbb{R}^{+}\right)$and $\Omega$ satisfy a Lipschitz condition of positive order on $S^{n-1}$, then $S$ is bounded on $L^{p}$ for $1<p<\infty$. Namazi [3] improved this result by replacing the Lipschitz condition by the condition that $\Omega \in L^{q}\left(S^{n-1}\right)$ for some $q>1$. In [4], Duoandikoetxea and Rubio de Francia developed some methods that can be used to study mapping properties of several kinds of operators in harmonic analysis, where they proved that $S$ is bounded on $L^{p}$ for $1<p<\infty$ when $h \in \Delta_{2}\left(\mathbb{R}^{+}\right)$and $\Omega \in L^{q}\left(S^{n-1}\right)$. In [5], Al-Salman and Pan proved that $S$ is bounded on $L^{p}$ for $1<p<\infty$ when $h \in \Delta_{\mathrm{s}}\left(\mathbb{R}^{+}\right)$ $(s>1)$ and $\Omega \in L \log L\left(S^{n-1}\right)$. Recently, using a method called Yano's extrapolation method [6,7], Sato [8] proved that $S$ extends to be an operator bounded on $L^{p}$ for $1<p$ $<\infty$ where $\Omega \in L \log L\left(S^{n-1}\right)$ and the radial function $h$ satisfying a rougher condition as a log type.

Define the function spaces

$$
\mathcal{L}_{a}\left(\mathbb{R}^{+}\right)=\left\{h: h \text { be measurable functions on } \mathbb{R}^{+}, L_{a}(h)<\infty\right\},
$$

where

$$
L_{a}(h)=\sup _{j \in \mathbb{Z}} \int_{2^{j}}^{2^{j+1}}|h(r)|(\log (2+|h(r)|))^{a} \frac{d r}{r} .
$$

And define the function space

$$
\mathcal{N}_{a}\left(\mathbb{R}^{+}\right)=\left\{h: h \text { be measurable functions on } \mathbb{R}^{+}, N_{a}(h)<\infty\right\},
$$

where

$$
N_{a}(h)=\sum_{m \geq 1} m^{a} 2^{m} d_{m}(h),
$$

with $d_{m}(h)=\sup _{k \in Z} 2^{-k}|E(k, m)|$ and $E(k, m)=\left\{r \in\left(2^{k}, 2^{k+1}\right]: 2^{m-1}<|h(r)| \leq 2^{m}\right\}$ for $m \geq 2, E(k, 1)=\left\{r \in\left(2^{k}, 2^{k+1}\right]:|h(r)| \leq 2\right\}$. Indeed, it is noted that for any $a>0$, $\mathcal{N}_{a}\left(\mathbb{R}^{+}\right) \subset \mathcal{L}_{a}\left(\mathbb{R}^{+}\right)$and $\mathcal{L}_{a+b}\left(\mathbb{R}^{+}\right) \subset \mathcal{N}_{a}\left(\mathbb{R}^{+}\right)$for some $b>1$.

Sato's main result is the following theorem:

Theorem A. [8] Suppose $\Omega$ is a function in $L \log L\left(S^{n-1}\right)$ satisfying (1.1) and $\|S(f)\|_{L^{p}\left(\mathbb{R}^{n}\right)} \leq C\|f\|_{L^{p}\left(\mathbb{R}^{n}\right)}\left(\right.$ or $h \in \mathcal{L}_{a}\left(\mathbb{R}^{+}\right)$for some $\left.a>2\right)$. Let $S$ be as in (1.2). Then, 
there is a constant $C$ such that

$$
\|S(f)\|_{L^{p}\left(\mathbb{R}^{n}\right)} \leq C\|f\|_{L^{p}\left(\mathbb{R}^{n}\right)}
$$

for all $p \in(1, \infty)$.

For the one-parameter case, there are also several other papers. Especially, in $[9,10]$, weighted $L^{p}$ boundedness of singular integrals was discussed. The reader also can refer to [11-13] for more background materials.

In the article, we mainly consider the $L^{p}$ boundedness for the multiple singular integrals with rough kernels. Suppose that $S^{d-1}(d=n$ or $m)$ is the unit sphere of $\mathbb{R}^{d}(d \geq$ 2) equipped with the usual Lebesgue measure $\mathrm{d} \sigma$. Let $\Omega \in L^{1}\left(S^{n-1} \times S^{m-1}\right)$ satisfy the following double cancelation condition:

$$
\int_{S^{n-1}} \Omega(u, v) \mathrm{d} \sigma(u)=0 \text { and } \int_{S^{m-1}} \Omega(u, v) \mathrm{d} \sigma(v)=0 .
$$

For $\alpha \geq 1$,

$$
\Delta_{\alpha}\left(\mathbb{R}^{+} \times \mathbb{R}^{+}\right)=\left\{h: h \text { be measurable functions on } \mathbb{R}^{+} \times \mathbb{R}^{+}, \quad\|h\|_{\Delta_{\alpha}}<\infty\right\},
$$

where

$$
\|h\|_{\Delta_{\alpha}}=\sup _{k, j \in \mathbb{Z}}\left(\int_{2^{k}}^{2^{k+1}} \int_{2^{j}}^{2^{j+1}}|h(r, s)|^{\alpha} \frac{\mathrm{d} r \mathrm{~d} s}{r s}\right)^{\frac{1}{\alpha}} .
$$

The multiple singular integral on the product space $\mathbb{R}^{n} \times \mathbb{R}^{m}$ is defined by the following form:

$$
T f\left(x_{1}, x_{2}\right)=\text { p.v. } \int_{\mathbb{R}^{n} \times \mathbb{R}^{m}} f\left(x_{1}-y_{1}, x_{2}-y_{2}\right) K\left(y_{1}, y_{2}\right) \mathrm{d} y_{1} \mathrm{~d} y_{2}
$$

for an appropriate function $f$ on $\mathbb{R}^{n} \times \mathbb{R}^{m}$, where

$$
K\left(y_{1}, y_{2}\right)=\left|y_{1}\right|^{-n}\left|y_{2}\right|^{-m} \Omega\left(y_{1}^{\prime}, y_{2}^{\prime}\right) h\left(\left|y_{1}\right|,\left|y_{2}\right|\right) .
$$

Let $L(\log L)^{\alpha}\left(S^{n-1} \times S^{m-1}\right)$ denote the class of the functions $\Omega$ defined on $S^{n-1} \times S^{m-1}$ satisfying the Zygmund condition: for $\alpha>0$,

$$
\int_{S^{n-1} \times S^{m-1}}|\Omega(\theta, \omega)|(\log (2+|\Omega(\theta, \omega)|))^{\alpha} \mathrm{d} \sigma(\theta) \mathrm{d} \sigma(\omega)<\infty .
$$

Historically, multiple singular integral was introduced by R. Fefferman and Stein's famous work on multiparameter harmonic analysis. Fefferman and Stein [14] proved that when $h \equiv 1, T$ is bounded on $L^{p}\left(\mathbb{R}^{n} \times \mathbb{R}^{m}\right)$ for $1<p<\infty$ if $\Omega$ satisfy certain smooth conditions. Their method mainly relies on so-called square function method. Subsequently, in [15], Duoandikoetxea used the method established in [4] and proved that $T$ is bounded on $L^{p}\left(\mathbb{R}^{n} \times \mathbb{R}^{m}\right)$ for $1<p<\infty$ when $\Omega \in L^{q}\left(S^{n-1} \times S^{m-1}\right)$ for some $q>1$ and $h \in \Delta_{2}\left(\mathbb{R}^{+} \times \mathbb{R}^{+}\right)$. In [16], Fan-Guo-Pan proved that $T$ is bounded on $L^{p}\left(\mathbb{R}^{n} \times \mathbb{R}^{m}\right)$ for $1<p<\infty$ for the case when $\Omega$ belongs to certain block spaces that contain $L^{q}\left(S^{n-1} \times S^{m-1}\right)$ (for $p=2$, it was proved by Jiang and Lu in [17] ) and $h=1$. In [18], Chen proved that $T$ is bounded on $L^{p}$ $\left(\mathbb{R}^{n} \times \mathbb{R}^{m}\right)$ for $1<p<\infty$ when $\Omega \in L(\log L)^{2}\left(S^{n-1} \times S^{m-1}\right)$ and $h=1$ where he mainly relies 
on the method of rotation. In [19], Al-Salman, Al-Qassem and Pan proved that $T$ is bounded on $L^{p}\left(\mathbb{R}^{n} \times \mathbb{R}^{m}\right)$ for $1<p<\infty$ when $\Omega \in L(\log L)^{2}\left(S^{n-1} \times S^{m-1}\right)$ and $h \in \Delta_{\alpha}$ for some $\alpha>1$, where their technique mostly based on refining the Duoandikoetxea-Rubia's Fourier transform estimates and Littlewood-paley theory. In the same paper, they also pointed out that for any $\varepsilon>0$, there is a function $\Omega \in L(\log L)^{2-\varepsilon}\left(S^{n-1} \times S^{m-1}\right)$ such that $T$ may fail to be bounded on $L^{p}\left(\mathbb{R}^{n} \times \mathbb{R}^{m}\right)$.

The main purpose of this paper is to improve the above results, especially the rough product radial part. For this reason, we introduce several measurable function spaces defined on $\mathbb{R}^{+} \times \mathbb{R}^{+}: \widetilde{\Delta}_{\alpha}\left(\mathbb{R}^{+} \times \mathbb{R}^{+}\right), \mathcal{L}_{\alpha}\left(\mathbb{R}^{+} \times \mathbb{R}^{+}\right)$and $\mathcal{N}_{\alpha}\left(\mathbb{R}^{+} \times \mathbb{R}^{+}\right)$for $\alpha>0$, where these spaces are equipped with the following "norms":

$$
\begin{gathered}
\|h\|_{\tilde{\Delta}_{\alpha}\left(\mathbb{R}^{+} \times \mathbb{R}^{+}\right)}=\sup _{k \in \mathbb{Z}}\left(\int_{2^{k}}^{2^{k+1}} \sup _{j \in \mathbb{Z}} \int_{2^{j}}^{2^{j+1}}|h(r, s)|^{\alpha} \frac{\mathrm{d} s}{s} \frac{\mathrm{d} r}{r}\right)^{\frac{1}{\alpha}}+\sup _{j \in \mathbb{Z}}\left(\int_{2^{j}}^{2^{j+1}} \sup _{k \in \mathbb{Z}} \int_{2^{k}}^{2^{k+1}}|h(r, s)|^{\alpha} \frac{\mathrm{d} r}{r} \frac{\mathrm{d} s}{s}\right)^{\frac{1}{\alpha}}, \\
\|h\|_{\mathcal{L}_{\alpha}\left(\mathbb{R}^{+} \times \mathbb{R}^{+}\right)}=\sup _{j, k \in \mathbb{Z}} \int_{2^{j}}^{2^{j+1} \int_{2^{k}}^{k^{k+1}}}|h(r, s)|(\log (2+|h(r, s)|))^{\alpha} \frac{\mathrm{d} r \mathrm{~d} s}{r s}, \\
\|h\| \mathcal{N}_{\alpha\left(\mathbb{R}^{+} \times \mathbb{R}^{+}\right)}=\sum_{m \geq 1} m^{a} 2^{m} D_{m}(h),
\end{gathered}
$$

with $D_{m}(h)=\sup _{k, j \in \mathbb{Z}} 2^{-k} 2^{-j}|E(k, j, m)|$ and $E(k, j, m)=\left\{(r, s) \in\left(2^{k}, 2^{k+1}\right] \times\left(2^{j}, 2^{j+1}\right]\right.$ : $\left.2^{m-1}<|h(r, s)| \leq 2^{m}\right\}$ for $m \geq 2, E(k, j, 1)=\left\{(r, s) \in\left(2^{k}, 2^{k+1}\right] \times\left(2^{j}, 2^{j+1}\right]:|h(r, s)| \leq 2\right\}$.

Remark 1.1. Of course by the usual modification, $\Delta_{\infty}\left(\mathbb{R}^{+} \times \mathbb{R}^{+}\right)=\widetilde{\Delta}_{\infty}\left(\mathbb{R}^{+} \times \mathbb{R}^{+}\right)=L^{\infty}\left(\mathbb{R}^{+} \times \mathbb{R}^{+}\right)$. For simplicity, we let $\Delta_{\alpha}=\Delta_{\alpha}\left(\mathbb{R}^{+} \times \mathbb{R}^{+}\right), \widetilde{\Delta}_{\alpha}=\widetilde{\Delta}_{\alpha}\left(\mathbb{R}^{+} \times \mathbb{R}^{+}\right), \mathcal{L}_{\alpha}=\mathcal{L}_{\alpha}\left(\mathbb{R}^{+} \times \mathbb{R}^{+}\right)$and $\mathcal{N}_{\alpha}=\mathcal{N}_{\alpha}\left(\mathbb{R}^{+} \times \mathbb{R}^{+}\right)$. It is easy to check that (1) $\Delta_{\infty} \subset \widetilde{\Delta}_{\alpha} \subset \Delta_{\alpha}$; (2) $\widetilde{\Delta}_{\alpha} \subset \widetilde{\Delta}_{\beta}$ if $1 \leq \beta<\alpha$; (3) for any $\alpha>0$, $\mathcal{N}_{\alpha} \subset \mathcal{L}_{\alpha}$ and $\mathcal{L}_{\alpha+\beta} \subset \mathcal{N}_{\alpha}$ for any $\beta>1$; (4) for any $\alpha>1$ and $\beta>0, \Delta_{\alpha} \subset \mathcal{L}_{\beta} \subset \Delta_{1}$.

Our main results are the following theorems:

Theorem 1.1. Suppose that $\Omega \in L(\log L)^{2}\left(S^{n-1} \times S^{m-1}\right)$ satisfying (1.3),

(1) if $h \in \mathcal{N}_{2}$ or $\mathcal{L}_{\alpha}$ for some $\alpha>3$, then there is a constant $C$ such that

$$
\|T f\|_{L^{2}\left(\mathbb{R}^{n} \times \mathbb{R}^{m}\right)} \leq C \mid f f \|_{L^{2}\left(\mathbb{R}^{n} \times \mathbb{R}^{m}\right)} .
$$

(2) if $h \in \widetilde{\Delta}_{\alpha}(\alpha \in(1,2])$, then there is a constant $C$, which is independent of $\alpha$, such that

$$
\|T f\|_{L^{p}\left(\mathbb{R}^{n} \times \mathbb{R}^{m}\right)} \leq\left. C \frac{1}{(\alpha-1)^{2}}\|h\|\right|_{\widetilde{\Delta}_{\alpha}}\|f\|_{L^{p}\left(\mathbb{R}^{n} \times \mathbb{R}^{m}\right)}
$$

for $p \in(1, \infty)$.

Remark 1.2. In [19], it was proved that $h \in \Delta_{\alpha}$ for some $\alpha>1$ and $\Omega \in L(\log L)^{2}\left(S^{n-1}\right.$ $\times S^{m-1}$ ) are sufficient for $L^{p}$ boundedness for the multiple singular integral $T$. As for $p=$ 2 , Theorem 1.1 extended this result. For $p \neq 2$, our condition $h \in \widetilde{\Delta}_{\alpha}(\alpha \in(1,2])$ is strong. However, our result gives a sharp constant estimate, which gives the following corollary when the product radial part is separated (that is, $h(r, s)=h_{1}(r) \cdot h_{2}(s)$ ).

Corollary 1.1. Suppose that $\Omega \in L(\log L)^{2}\left(S^{n-1} \times S^{m-1}\right)$ satisfying (1.3) and if $h(r, s)=$ $h_{1}(r) \cdot h_{2}(s)$, where $h_{1}$ or $h_{2}$ satisfies one of the following case: 
(1) $h_{1} \in \Delta_{\alpha}\left(\mathbb{R}^{+}\right)((\alpha>1))$ and $h_{2} \in \mathcal{N}_{2}\left(\mathbb{R}^{+}\right)\left(\right.$or $h_{2} \in \mathcal{L}_{a}\left(\mathbb{R}^{+}\right)$for some $\left.a>3\right)$;

(2) $h_{1} \in \mathcal{N}_{2}\left(\mathbb{R}^{+}\right)\left(\right.$or $h_{1} \in \mathcal{L}_{a}\left(\mathbb{R}^{+}\right)$fsor some $\left.a>3\right)$ and $h_{2} \in \Delta_{s}\left(\mathbb{R}^{+}\right)((s>1))$,

then there is a constant $C$ such that

$$
\|T f\|_{L^{p}\left(\mathbb{R}^{n} \times \mathbb{R}^{m}\right)} \leq C|| f \|_{L^{p}\left(\mathbb{R}^{n} \times \mathbb{R}^{m}\right)}
$$

for $p \in(1, \infty)$.

Our proof of the above theorem is based on the argument of Sato [8], which mainly relied on Yano's extrapolation method. The following theorem is the key step to prove Theorem 1.1.

Theorem 1.2. Suppose that $\Omega \in L^{q}\left(S^{n-1} \times S^{m-1}\right)(q \in(1,2])$ satisfying (1.3).

(1) If $h \in \Delta_{\alpha}(\alpha \in(1,2])$, then there exists a constant $C$, which is independent of $q$, $\alpha, \Omega, h$, such that

$$
\|T f\|_{L^{2}\left(\mathbb{R}^{n} \times \mathbb{R}^{m}\right)} \leq C \frac{1}{(\alpha-1)^{2}} \frac{1}{(q-1)^{2}}\|h\|_{\Delta_{\alpha}}\|\Omega\|_{L^{p}\left(S^{n-1} \times S^{m-1}\right)}\|f\|_{L^{2}\left(\mathbb{R}^{n} \times \mathbb{R}^{m}\right)} .
$$

(2) If $h \in \widetilde{\Delta}_{\alpha}(\alpha \in(1,2])$, then there exists a constant $C$, which is independent of $q$, $\alpha, \Omega, h$, such that

$$
\|T f\|_{L^{p}\left(\mathbb{R}^{n} \times \mathbb{R}^{m}\right)} \leq C \frac{1}{(\alpha-1)^{2}} \frac{1}{(q-1)^{2}}\|h\|_{\widetilde{\Delta}_{\alpha}}\|\Omega\|_{L^{q}\left(S^{n-1} \times S^{m-1}\right)}\|f\|_{L^{p}\left(\mathbb{R}^{n} \times \mathbb{R}^{m}\right)}
$$

for $p \in(1, \infty)$.

Remark 1.3. Corollary 1 in [15] asserted that if $h \in \Delta_{2}$ and $\Omega \in L^{q}(q>1)\left(S^{n-1} \times S^{m-}\right.$ $\left.{ }^{1}\right)$, then $T$ is bounded in $L^{p}\left(\mathbb{R}^{n} \times \mathbb{R}^{m}\right)$ for $p>1$. After a careful check of its proof, we find that the condition $h \in \Delta_{2}$ is not sufficient for $p \neq 2$ since the two partial maximal functions are taken supremum both $j$ and $k$, it seems that if $h \in \Delta_{2}$, the partial maximal function is not pointwise controlled by the one-parameter maximal function case (line 10-13, [15]). If we substitute $h \in \Delta_{2}$ with $h \in \widetilde{\Delta}_{2}$, Corollary 1 in [15] is corrected. This is why we introduce the space $\widetilde{\Delta}_{\alpha}$. Of course, we remark that our result is mainly influenced by the idea and the technique established in [15]: Littlewood-Paley theory for product theory, Fourier transform estimates, etc.

Remark 1.4. The maximal multiple singular integral is defined as

$$
T^{*} f\left(x_{1}, x_{2}\right)=\sup _{\varepsilon_{1}>0, \varepsilon_{2}>0}\left|\iint_{\left|y_{1}\right| \geq \varepsilon_{1},\left|y_{2}\right| \geq \varepsilon_{2}} f\left(x_{1}-y_{1}, x_{2}-y_{2}\right) K\left(y_{1}, y_{2}\right) \mathrm{d} y_{1} \mathrm{~d} y_{2}\right|,
$$

where $K$ is as in (1.5). By the estimates we have established and Yano's extrapolation method, combining with [20], we have the same result for the maximal multiple singular integral as in [19]:

Theorem 1.3. Suppose that $\Omega \in L(\log L)^{2}\left(S^{n-1} \times S^{m-1}\right)$ satisfies (1.3) and $h \in \Delta_{\infty}$, then there exists a constant $C$, such that

$$
\left\|T^{*} f\right\|_{L^{p}\left(\mathbb{R}^{n} \times \mathbb{R}^{m}\right)} \leq C \mid\|h\|_{\Delta_{\infty}}\|f\|_{L^{p}\left(\mathbb{R}^{n} \times \mathbb{R}^{m}\right)},
$$


for $p \in(1, \infty)$.

We leave the proof to the interested reader. But we do not know whether $h$ can be extended to more general case like $\widetilde{\Delta}_{\alpha}(1<\alpha<\infty)$.

This paper is organized as the following. In Section 2, we give the proof of Theorem 1.2. In Section 3, we give the proof of Theorem 1.1 and Corollary 1.1. Throughout this paper, the letter $C$ will stand for a constant that may vary at each occurrence but that is independent of the essential variables and $p^{\prime}$ be the conjugation of $p$ satisfying $\frac{1}{p}+\frac{1}{p^{\prime}}=1$.

\section{Proof of Theorem $\mathbf{1 . 2}$}

Let $\Omega, h$ be as in Theorem 1.2. We let $\rho \geq 2$, define

$$
E_{k, j}=\left\{\left(y_{1}, y_{2}\right) \in \mathbb{R}^{n+m}: \rho^{k}<\left|y_{1}\right| \leq \rho^{k+1}, \rho^{j}<\left|y_{2}\right| \leq \rho^{j+1}\right\}
$$

and measures $\sigma_{k, j}$ by

$$
\sigma_{k, j} * f\left(x_{1}, x_{2}\right)=\iint_{E_{k, j}} K\left(y_{1}, y_{2}\right) f\left(x_{1}-y_{1}, x_{2}-y_{2}\right) \mathrm{d} y_{1} \mathrm{~d} y_{2}
$$

So

$$
T f=\sum_{k, j} \sigma_{k, j} * f
$$

Define $\sigma^{*}$ by $\sigma^{*} f(x)=\sup _{k, j}|| \sigma_{k, j}|* f(x)|$, where $\left|\sigma_{k, j}\right|$ denotes the total variation. Let $\mu_{k, j}=\left|\sigma_{k, j}\right|$ and define $\mu^{*}$ by $\mu^{*} f(x)=\sup _{k, j}\left|\mu_{k, j} * f(x)\right|$. Let $\theta \in(0,1), \delta(p)=\mid 1 / p-1 /$ $p^{\prime} \mid$, we have the following two lemmas.

Lemma 2.1. For $p>1+\theta$, suppose that $\Omega \in L^{q}\left(S^{n-1} \times S^{m-1}\right)(q \in(1,2])$ satisfying (1.3) and $h \in \widetilde{\Delta}_{\alpha}(\alpha \in(1,2])$, we have

$$
\left\|\mu^{*} f\right\|_{L^{p}\left(\mathbb{R}^{n} \times \mathbb{R}^{m}\right)} \leq \operatorname{Clog}^{2} \rho\|\Omega\|_{L^{q}\left(S^{n-1} \times S^{m-1}\right)}\|h\|_{\widetilde{\Delta}_{\alpha}}\left(1-2^{-\frac{\theta}{2}}\right)^{-2.2 / p}\|f\|_{L^{p}\left(\mathbb{R}^{n} \times \mathbb{R}^{m}\right)}(2
$$

where the constant $C$ is independent of $q, \alpha, \Omega, h$.

Lemma 2.2. (1). Suppose that $\Omega \in L^{q}\left(S^{n-1} \times S^{m-1}\right)(q \in(1,2])$ satisfying (1.3) and $h \in$ $\Delta_{\alpha}(\alpha \in(1,2])$,

$$
\|T f\|_{L^{2}\left(\mathbb{R}^{n} \times \mathbb{R}^{m}\right)} \leq C \log ^{2} \rho\|\Omega\|_{L^{q}\left(S^{n-1} \times S^{m-1}\right)}\|h\|_{\Delta_{\alpha}}\|f\|_{L^{2}\left(\mathbb{R}^{n} \times \mathbb{R}^{m}\right)}
$$

(2). For $p \in(1+\theta,(1+\theta) / \theta)$, suppose that $\Omega \in L^{q}\left(S^{n-1} \times S^{m-1}\right)(q \in(1,2])$ satisfying (1.3) and $h \in \widetilde{\Delta}_{\alpha}(\alpha \in(1,2])$, we have

$$
\|T f\|_{L^{p}\left(\mathbb{R}^{n} \times \mathbb{R}^{m}\right)} \leq C \log ^{2} \rho\|\Omega\|_{L^{q}\left(S^{n-1} \times S^{m-1}\right)}\|h\|_{\widetilde{\Delta}_{\alpha}}\left(1-2^{-\frac{\theta}{2}}\right)^{-2(1+\delta(p))}\|f\|_{L^{p}\left(\mathbb{R}^{n} \times \mathbb{R}^{m}\right),}
$$

where the constant $C$ is independent of $q, \alpha, \Omega, h$.

If Lemma 2.2 is proved, since $\theta \in(0,1)$ is arbitrary and we choose $\rho=2^{q^{\prime} \alpha^{\prime}}$, then Theorem 1.2 is an immediate consequence of Lemma 2.2 immediately.

Now, we prove part (1) of Lemma 2.2. For simplicity, we let $A=\log ^{2} \rho\|\Omega\|_{L^{q}\left(S^{n-1} \times S^{m-1}\right)}\|h\|_{\Delta_{\alpha^{*}}}$. Firstly, we have the following estimates for the measures $\sigma_{k, j}$ : 


$$
\begin{aligned}
& \left\|\sigma_{k, j}\right\| \leq c_{1} A \\
& \left.\left|\hat{\sigma}_{k, j}\left(\xi_{1}, \xi_{2}\right) \leq c_{2} A\right| \rho^{k} \xi_{1}\right|^{ \pm \frac{1}{2 q^{\prime} \alpha^{\prime}}}\left|\rho^{j} \xi_{2}\right|^{ \pm \frac{1}{2 q^{\prime} \alpha^{\prime}}}
\end{aligned}
$$

for some constants $c_{i}$. The equation (2.4) is the consequence of the following result:

$$
\begin{aligned}
& \left\|\sigma_{k, j}\right\|=\left|\sigma_{k, j}\right|\left(\mathbb{R}^{n} \times \mathbb{R}^{m}\right) \\
& \leq \int_{\rho^{k}}^{\rho^{k+1}} \int_{\rho^{j}}^{\rho^{j+1}} \int_{S^{n-1} \times S^{m-1}}|\Omega(u, v) \| h(r, s)| \mathrm{d} \sigma(u) \mathrm{d} \sigma(v) \frac{\mathrm{d} r \mathrm{~d} s}{r s} \\
& \leq C \log ^{2} \rho\|\Omega\|_{L^{1}\left(S^{n-1} \times S^{m-1}\right)}\|h\|_{\Delta_{1}} .
\end{aligned}
$$

Now, we turn to prove (2.5), note

$$
\hat{\sigma}_{k, j}\left(\xi_{1}, \xi_{2}\right)=\int_{\rho^{k}}^{\rho^{k+1}} \int_{\rho^{j}}^{j+1} \int_{S^{n-1} \times S^{m-1}} \Omega(u, v) h(r, s) e^{-2 \pi i\left(\xi_{1} \cdot r u+\xi_{2} \cdot s v\right)} \mathrm{d} \sigma(u) \mathrm{d} \sigma(v) \frac{\mathrm{d} r \mathrm{~d} s}{r s}
$$

and we define

$$
F\left(r, s, \xi_{1}, \xi_{2}\right)=\int_{S^{n-1} \times S^{m-1}} \Omega(u, v) e^{-2 \pi i\left(\xi_{1} \cdot r u+\xi_{2} \cdot s v\right)} \mathrm{d} \sigma(u) \mathrm{d} \sigma(v) .
$$

Then, by Hölder's inequality,

$$
\begin{aligned}
& \left|\hat{\sigma}_{k, j}\left(\xi_{1}, \xi_{2}\right)\right|=\left|\int_{\rho^{k}}^{\rho^{k+1}} \int_{\rho^{j}}^{j+1} F\left(r, s, \xi_{1}, \xi_{2}\right) h(r, s) \frac{\mathrm{d} r \mathrm{~d} s}{r s}\right| \\
& \leq\left(\int_{\rho^{k}}^{\rho^{k+1}} \int_{\rho^{j}}^{\rho^{j+1}}|h(r, s)|^{\alpha} \frac{\mathrm{d} r \mathrm{~d} s}{r s}\right)^{\frac{1}{\alpha}}\left(\int_{\rho^{k}}^{\rho^{k+1}} \int_{\rho^{j}}^{\rho^{j+1}}\left|F\left(r, s, \xi_{1}, \xi_{2}\right)\right|^{\alpha^{\prime}} \frac{\mathrm{d} r \mathrm{~d} s}{r s}\right)^{\frac{1}{\alpha^{\prime}}} \\
& \leq\left(\int_{\rho^{k}}^{\rho^{k+1}} \int_{\rho^{j}}^{\rho^{j+1}}|h(r, s)|^{\alpha} \frac{\mathrm{d} r \mathrm{~d} s}{r s}\right)^{\frac{1}{\alpha}}\|\Omega\|_{L^{\prime}\left(S^{n-1} \times S^{m-1}\right)}^{\frac{\alpha^{\prime}-2}{\alpha^{\prime}}}\left(\int_{\rho^{k}}^{\rho^{k+1}} \int_{\rho^{j}}^{\rho^{j+1}}\left|F\left(r, s, \xi_{1}, \xi_{2}\right)\right|^{2} \frac{\mathrm{d} r \mathrm{~d} s}{r s}\right)^{\frac{1}{\alpha^{\prime}}}
\end{aligned}
$$

while here

$$
\begin{aligned}
& \int_{\rho^{k}}^{\rho^{k+1}} \int_{\rho^{j}}^{j+1}\left|F\left(r, s, \xi_{1}, \xi_{2}\right)\right|^{2} \frac{\mathrm{d} r \mathrm{~d} s}{r s} \\
& =\int_{\rho^{k}}^{\rho^{k+1}} \int_{\rho^{j}}^{\rho^{j+1}} \iint_{\left(S^{n-1} \times S^{m-1}\right)^{2}} \Omega(u, v) \overline{\Omega\left(u^{\prime}, v^{\prime}\right)} e^{-2 \pi i\left(\xi_{1} \cdot r \cdot r\left(u-u^{\prime}\right)+\xi_{2} \cdot s\left(v-v^{\prime}\right)\right)} \mathrm{d} \sigma\left(u^{\prime}\right) \mathrm{d} \sigma(v) \mathrm{d} \sigma\left(v^{\prime}\right) \frac{\mathrm{d} r \mathrm{~d} s}{r s} \\
& \leq \iint_{\left(S^{n-1} \times S^{m-1}\right)^{2}} \Omega(u, v) \overline{\Omega\left(u^{\prime}, v^{\prime}\right)} \int_{\rho^{k}}^{\rho^{k+1}} \int_{\rho^{j}}^{\rho^{j+1}} e^{-2 \pi i\left(\xi_{1} \cdot r\left(u-u^{\prime}\right)+\xi_{\xi} \cdot s\left(v-v^{\prime}\right)\right)} \frac{\mathrm{d} r \mathrm{~d} s}{r s} \mathrm{~d} \sigma(u) \mathrm{d} \sigma\left(u^{\prime}\right) \mathrm{d} \sigma(v) \mathrm{d} \sigma\left(v^{\prime}\right) \\
& \leq C \log ^{2} \rho\|\Omega\|_{L^{q}\left(S^{n-1} \times S^{m-1}\right)}^{2}\left|\rho^{k} \xi_{1}\right|^{-\varepsilon}\left|\rho^{j} \xi_{2}\right|^{-\varepsilon} \\
& \left(\int_{s^{n-1} \times S^{n-1}} \frac{\mathrm{d} \sigma(u) \mathrm{d} \sigma\left(u^{\prime}\right)}{\left|\xi^{\prime}{ }_{1} \cdot\left(u-u^{\prime}\right)\right|^{\mid q^{\prime}}}\right)^{\frac{1}{q^{\prime}}}\left(\int_{S^{m-1} \times S^{m-1}} \frac{\mathrm{d} \sigma(v) \mathrm{d} \sigma\left(v^{\prime}\right)}{\left.\xi_{2}^{\prime} \cdot\left(v-v^{\prime}\right)\right|^{\varepsilon q^{\prime}}}\right)^{\frac{1}{q^{\prime}}} \text {. }
\end{aligned}
$$


When $\varepsilon q^{\prime}<1$ (indeed we set $\varepsilon=\frac{1}{2 q^{\prime}}$ ), the integrals $\left(\int_{s^{n-1} \times S^{n-1}} \frac{\mathrm{d} \sigma(u) \mathrm{d} \sigma\left(u^{\prime}\right)}{\left|\xi^{\prime}{ }_{1} \cdot\left(u-u^{\prime}\right)\right| \varepsilon q^{\prime}}\right)^{\frac{1}{\alpha^{\prime} q^{\prime}}}$ and $\left(\int_{S^{m-1} \times S^{m-1}} \frac{\mathrm{d} \sigma(v) \mathrm{d} \sigma\left(v^{\prime}\right)}{\left|\xi_{2}^{\prime} \cdot\left(v-v^{\prime}\right)\right|^{\varepsilon q^{\prime}}}\right) \frac{1}{\alpha^{\prime} q^{\prime}}$ are finite and independent of $q$ and $\alpha$. So we have

$$
\left|\hat{\sigma}_{k, j}\left(\xi_{1}, \xi_{2}\right)\right| \leq C \log ^{2} \rho|| h\left\|_{\Delta_{\alpha}}|| \Omega\right\|_{L^{q}\left(S^{n-1} \times S^{m-1}\right)}\left|\rho^{k} \xi_{1}\right|^{-\frac{1}{2 q^{\prime} \alpha^{\prime}}}\left|\rho^{j} \xi_{2}\right|^{-\frac{1}{2 q^{\prime} \alpha^{\prime}}} .
$$

Since $\Omega$ satisfies the condition (1.3), we have $\hat{\sigma}_{k, j}\left(0, \xi_{2}\right)=0$ and then $\left|\hat{\sigma}_{k, j}\left(\xi_{1}, \xi_{2}\right)\right|$ equals to

$$
\begin{aligned}
& \left|\hat{\sigma}_{k, j}\left(\xi_{1}, \xi_{2}\right)-\hat{\sigma}_{k, j}\left(0, \xi_{2}\right)\right| \\
& =\left|\int_{\rho^{k}}^{\rho^{k+1}} \int_{\rho^{j}}^{\rho^{j+1}} \int_{S^{n-1} \times S^{m-1}} \Omega(u, v) h(r, s)\left[e^{-2 \pi i \xi_{1} \cdot r u}-1\right] e^{-2 \pi i \xi_{2} \cdot s v} \mathrm{~d} \sigma(u) \mathrm{d} \sigma(v) \frac{\mathrm{d} r \mathrm{~d} s}{r s}\right| \\
& \leq \int_{\rho^{k}}^{\rho^{k+1}} \int_{S^{n-1}}\left|\int_{\rho^{j}}^{\rho^{j+1}} \int_{S^{m-1}} \Omega(u, v) h(r, s) e^{-2 \pi i \xi_{2} \cdot s v} d v \frac{\mathrm{d} s}{s}\right|\left|e^{-2 \pi i \xi_{1} \cdot r u}-1\right| d u \frac{\mathrm{d} r}{r} \\
& \leq \int_{\rho^{k}}^{\rho^{k+1}} \int_{S^{n-1}}\left|\int_{\rho^{j}}^{\rho^{j+1}} \int_{S^{m-1}} \Omega(u, v) h(r, s) e^{-2 \pi i \xi_{2} \cdot s v} d v \frac{\mathrm{d} s}{s}\right| \min \left(\left\{2, r\left|\xi_{1}\right|\right\}\right) d u \frac{\mathrm{d} r}{r} \\
& \leq \quad \operatorname{Cog}^{2} \rho|| h\left\|_{\Delta_{\alpha}}\right\| \Omega \|_{L^{q}\left(S^{n-1} \times S^{m-1}\right)}\left|\rho^{k} \xi_{1}\right|^{\frac{1}{2^{\prime} \alpha^{\prime}}}\left|\rho^{j} \xi_{2}\right|^{-\frac{1}{2 q^{\prime} \alpha^{\prime}}} .
\end{aligned}
$$

The same way as above, we have $\left|\hat{\sigma}_{k, j}\left(\xi_{1}, \xi_{2}\right)\right|$ equals to

$$
\left|\hat{\sigma}_{k, j}\left(\xi_{1}, \xi_{2}\right)-\hat{\sigma}_{k, j}\left(\xi_{1}, 0\right)\right| \leq C \log ^{2} \rho\|h\|_{\Delta_{\alpha}}|| \Omega \|_{L^{q}\left(S^{n-1} \times S^{m-1}\right)}\left|\rho^{k} \xi_{1}\right|^{-\frac{1}{2 q^{\prime} \alpha^{\prime}}}\left|\rho^{j} \xi_{2}\right|^{\frac{1}{2 q^{\prime} \alpha^{\prime}}} .
$$

Also we have $\left|\hat{\sigma}_{k, j}\left(\xi_{1}, \xi_{2}\right)\right|$ equals to

$$
\begin{aligned}
& \left|\hat{\sigma}_{k, j}\left(\xi_{1}, \xi_{2}\right)-\hat{\sigma}_{k, j}\left(\xi_{1}, 0\right)-\hat{\sigma}_{k, j}\left(0, \xi_{2}\right)+\hat{\sigma}_{k, j}(0,0)\right| \\
& \leq\left. C \log ^{2} \rho|| h\right|_{\Delta_{\alpha}}|| \Omega \|_{L^{q}\left(S^{n-1} \times S^{m-1}\right)}\left|\rho^{k} \xi_{1}\right|^{\frac{1}{2 q^{\prime} \alpha^{\prime}}}\left|\rho^{j} \xi_{2}\right|^{\frac{1}{2 q^{\prime} \alpha^{\prime}}} .
\end{aligned}
$$

Consequently, the inequality (2.5) is just the combination of (2.6), (2.7),(2.8) and (2.9).

Let $\psi^{1} \in \mathcal{S}\left(\mathbb{R}^{n}\right), \psi^{2} \in \mathcal{S}\left(\mathbb{R}^{m}\right)$, such that

$$
\begin{gathered}
\operatorname{supp}\left(\psi^{i}\left(\xi_{i}\right)\right) \subset\left\{\frac{1}{\rho} \leq\left|\xi_{i}\right|<\rho\right\}, i=1,2, \\
0 \leq \psi^{i}\left(\xi_{i}\right) \leq 1, i=1,2,
\end{gathered}
$$

and

$$
\sum_{k=-\infty}^{\infty}\left|\left(\psi^{1}\right)\left(\rho^{k} \xi_{1}\right)\right|^{2}=\sum_{j=-\infty}^{\infty}\left|\left(\psi^{2}\right)\left(\rho^{j} \xi_{2}\right)\right|^{2}=1
$$

Let $\psi_{k}^{1}, \psi_{j}^{2}$ as $\left(\psi_{k}^{1}\right)^{\Lambda}\left(\xi_{1}\right)=\psi^{1}\left(\rho^{k} \xi_{1}\right),\left(\psi_{j}^{2}\right)^{\Lambda}\left(\xi_{2}\right)=\psi^{2}\left(\rho^{j} \xi_{2}\right)$, respectively. Then, we have 


$$
\begin{aligned}
T f & =\sum_{k, j} \sigma_{k, j} * f \\
& =\sum_{k, j} \sum_{l, m} \sigma_{k, j} *\left(\psi_{k+1}^{1} \otimes \psi_{j+m}^{2}\right) *\left(\psi_{k+l}^{1} \otimes \psi_{j+m}^{2}\right) * f \\
& \triangleq \sum_{l, m} T_{l, m} f
\end{aligned}
$$

where

$$
T_{l, m} f=\sum_{k, j} \sigma_{k, j} *\left(\psi_{k+1}^{1} \otimes \psi_{j+m}^{2}\right) *\left(\psi_{k+l}^{1} \otimes \psi_{j+m}^{2}\right) * f .
$$

Then, by Plancherel's theorem and (2.5), we have

$$
\begin{aligned}
& \left\|T_{l, m} f\right\|_{L^{2}\left(\mathbb{R}^{n} \times \mathbb{R}^{m}\right)}^{2} \leq \sum_{k, j} C \iint_{D(k+l, j+m)}\left|\hat{\sigma}_{k, j}\left(\xi_{1}, \xi_{2}\right)\right|^{2}\left|\hat{f}\left(\xi_{1}, \xi_{2}\right)\right|^{2} \mathrm{~d} \xi_{1} \mathrm{~d} \xi_{2} \\
& \leq C A^{2} \min \left\{1, \rho^{-2 \frac{1}{2 q^{\prime} \alpha^{\prime}}(|l|-1)}\right\} \min \left\{1, \rho^{-2 \frac{1}{2 q^{\prime} \alpha^{\prime}}(|m|-1)}\right\} \sum_{k, j \in \mathbb{Z}(k+l, j+m)} \iint_{(k+j)}\left|\hat{f}\left(\xi_{1}, \xi_{2}\right)\right|^{2} \mathrm{~d} \xi_{1} \mathrm{~d} \xi_{2} \\
& \leq C A^{2} \min \left\{1, \rho^{-2 \frac{1}{2 q^{\prime} \alpha^{\prime}}(|l|-1)}\right\} \min \left\{1, \rho^{-2 \frac{1}{2 q^{\prime} \alpha^{\prime}}(|m|-1)}\right\}\|f\|_{L^{2}\left(\mathbb{R}^{n} \times \mathbb{R}^{m}\right)}^{2}
\end{aligned}
$$

where $D(k, j)=\left\{(\xi, \eta): \rho^{-k-1} \leq|\xi| \leq \rho^{-k+1}, \rho^{-j-1} \leq|\eta| \leq \rho^{-j+1}\right\}$. By above estimates and Minkowski's inequality, we give the proof of part (1).

Now, we turn to prove part (2) of Lemma 2.2, take for Lemma 2.1 is granted. We let $A^{\prime}=\log ^{2} \rho\|\Omega\|_{L^{q}\left(S^{n-1} \times S^{m-1}\right)}\|h\|_{\widetilde{\Delta}_{\alpha}}$ and $B=\left(1-2^{-\frac{\theta}{2}}\right)^{-2}$ for simplicity. We have

$$
\begin{aligned}
& || \sigma_{k, j} \| \leq c_{1} A^{\prime} \\
& \left|\hat{\sigma}_{k, j}\left(\xi_{1}, \xi_{2}\right)\right| \leq c_{2} A^{\prime}\left|\rho^{k} \xi_{1}\right|^{ \pm \frac{1}{2 q^{\prime} \alpha^{\prime}}\left|\rho^{j} \xi_{2}\right|^{ \pm} \frac{1}{2 q^{\prime} \alpha^{\prime}}} \\
& \left\|\sigma^{*}(f)\right\|_{L^{p}\left(\mathbb{R}^{n} \times \mathbb{R}^{m}\right)} \leq C_{p} A^{\prime} B^{\frac{2}{p}}\|f\|_{L^{p}\left(\mathbb{R}^{n} \times \mathbb{R}^{m}\right)} \text { for } p>1+\theta,
\end{aligned}
$$

for some constants $c_{i}$ and $C_{p}$. where Eqs. (2.12) and (2.13) follow (2.4) and (2.5), respectively, (2.14) is just (2.1).

Lemma 2.3. Let $u \in(1+\theta, 2]$, define a number $v$ by $\frac{1}{v}-\frac{1}{2}=\frac{1}{2 u}$. Then, we have the vector-valued inequality

$$
\left\|\left(\sum_{k, j}\left|\sigma_{k, j} * g_{k, j}\right|^{2}\right)^{\frac{1}{2}}\right\|_{L^{v}\left(\mathbb{R}^{n} \times \mathbb{R}^{m}\right)} \leq\left(c_{1} C_{u}\right)^{\frac{1}{2}} A^{\prime} B^{\frac{1}{u}}\left\|\left(\sum_{k, j}\left|g_{k, j}\right|^{2}\right)^{\frac{1}{2}}\right\|_{L^{v}\left(\mathbb{R}^{n} \times \mathbb{R}^{m}\right)},
$$

where $c_{1}$ and $C_{u}$ are as in (2.4) and (2.14), respectively.

Proof. The proof is the same way as in one parameter case, and we prove it here for completeness. 
Since

$$
\left\|\sum_{k, j}\left|\sigma_{k, j} * g_{k, j}\right|\right\|_{L^{1}\left(\mathbb{R}^{n} \times \mathbb{R}^{m}\right)} \leq c_{1} A^{\prime}\left\|\sum_{k, j}\left|g_{k, j}\right|\right\|_{L^{1}\left(\mathbb{R}^{n} \times \mathbb{R}^{m}\right)}
$$

and

$$
\left\|\sup _{k, j}\left|\sigma_{k, j} * g_{k, j}\right|\right\|_{L^{u}\left(\mathbb{R}^{n} \times \mathbb{R}^{m}\right)} \leq\left\|\sigma *\left(\sup _{k, j}\left|g_{k, j}\right|\right)\right\|_{L^{u}\left(\mathbb{R}^{n} \times \mathbb{R}^{m}\right)} \leq C_{u} A^{\prime} B^{\frac{2}{u}}\left\|\sup _{k, j}\left|g_{k, j}\right|\right\|_{L^{u}\left(\mathbb{R}^{n} \times \mathbb{R}^{m}\right)}
$$

Interpolation between the above two inequalities completed the proof of the lemma. By the Littlewood-Paley theory, we have

$$
\begin{aligned}
& \left\|T_{l, m} f\right\|_{L^{p}\left(\mathbb{R}^{n} \times \mathbb{R}^{m}\right)} \leq C_{p}\left\|\left(\sum_{k, j}\left|\sigma_{k, j} *\left(\psi_{k+l}^{1} \otimes \psi_{j+m}^{2}\right) * f\right|\right)^{1 / 2}\right\|_{L_{L^{p}\left(\mathbb{R}^{n} \times \mathbb{R}^{m}\right)^{\prime}}} \\
& \left\|\left(\sum_{k, j}\left|\left(\psi_{k+1}^{1} \otimes \psi_{j+m}^{2}\right) * f\right|\right)^{1 / 2}\right\|_{L^{p}\left(\mathbb{R}^{n} \times \mathbb{R}^{m}\right)} \leq C_{p}\|f\|_{L^{p}\left(\mathbb{R}^{n} \times \mathbb{R}^{m}\right)},
\end{aligned}
$$

where $p \in(1, \infty)$ and $C_{p}$ is independent of $\rho$. Suppose that $1+\theta \leq p \leq \frac{4}{3-\theta}$. Then, we can find $u \in(1+\theta, 2]$ such that $\frac{1}{p}=\frac{1}{2}+\frac{1-\theta}{2 u}$. Let $v: \frac{1}{v}=\frac{1}{2}+\frac{1}{2 u}$, by Lemma 2.3, (2.15) and (2.16), we have

$$
\left\|T_{l, m} f\right\|_{v} \leq C A^{\prime} B^{\frac{1}{u}}\|f\|_{v}
$$

Since $\frac{1}{p}=\frac{1-\theta}{v}+\frac{\theta}{2}$, by interpolation, we have

$$
\left\|T_{l, m} f\right\|_{L^{p}\left(\mathbb{R}^{n} \times \mathbb{R}^{m}\right)} \leq C A^{\prime} B^{\frac{1-\theta}{u}} \min \left\{1, \rho^{-\frac{\theta}{2 q^{\prime} \alpha^{\prime}}(|m|-1)}\right\}\|f\|_{L^{p}\left(\mathbb{R}^{n} \times \mathbb{R}^{m}\right)}
$$

Then

$$
\|T f\|_{L^{p}\left(\mathbb{R}^{n} \times \mathbb{R}^{m}\right)} \leq \sum_{l, m}\left\|T_{l, m} f\right\|_{p} \leq C A^{\prime} B^{\frac{1-\theta}{u}}\left(1-\rho^{-\frac{\theta}{2 q^{\prime} \alpha^{\prime}}}\right)^{-2}\|f\|_{L^{p}\left(\mathbb{R}^{n} \times \mathbb{R}^{m}\right)} .
$$

Since $\rho=2^{q^{\prime} \alpha^{\prime}}, B=\left(1-2^{-\frac{\theta}{2}}\right)^{-2}$ and $\frac{1-\theta}{u}+1=\frac{2}{p}$, then we have

$$
\|T f\|_{L^{p}\left(\mathbb{R}^{n} \times \mathbb{R}^{m}\right)} \leq C A^{\prime} B^{\frac{2}{p}}\|f\|_{L^{p}\left(\mathbb{R}^{n} \times \mathbb{R}^{m}\right)} .
$$

When $p=2$, by Eq. (2.11) and $B>\left(1-2^{-\frac{1}{2}}\right)^{-2}$, we have

$$
\|T f\|_{L^{2}\left(\mathbb{R}^{n} \times \mathbb{R}^{m}\right)} \leq \sum_{l, m}\left\|T_{l, m} f\right\|_{L^{2}\left(\mathbb{R}^{n} \times \mathbb{R}^{m}\right)} \leq C A^{\prime} B\|f\|_{L^{2}\left(\mathbb{R}^{n} \times \mathbb{R}^{m}\right)} .
$$

By duality and interpolation, we can now finish the proof of Lemma 2.2.

Now, we give a proof of Lemma 2.1. Since $\left\|\mu^{*} f\right\|_{\infty} \leq c_{1} A|| f \mid \|_{\infty}$, by taking into account an interpolation, it suffices to prove $(2.1)$ for $p \in(1+\theta, 2]$. We recall that $\mu_{k, j}=\left|\sigma_{k, j}\right|$ and $\mu^{*} f(x)=\sup _{k, j}\left|\mu_{k, j} * f(x)\right|$. The following four estimates for $\mu_{k, j}$ are similar with the equations (2.4) and (2.5): 


$$
\begin{aligned}
& || \mu_{k, j}|| \leq A^{\prime} \\
& \left|\hat{\mu}_{k, j}\left(\xi_{1}, \xi_{2}\right)-\hat{\mu}_{k, j}\left(0, \xi_{2}\right)\right| \leq C A^{\prime}\left|\rho^{k} \xi_{1}\right|^{\frac{1}{2 q^{\prime} \alpha^{\prime}}}\left|\rho^{j} \xi_{2}\right|^{-\frac{1}{2 q^{\prime} \alpha^{\prime}}} \\
& \left|\hat{\mu}_{k, j}\left(\xi_{1}, \xi_{2}\right)-\hat{\mu}_{k, j}\left(\xi_{1}, 0\right)\right| \leq C A^{\prime}\left|\rho^{k} \xi_{1}\right|^{-\frac{1}{2 q^{\prime} \alpha^{\prime}}}\left|\rho^{j} \xi_{2}\right|^{\frac{1}{2 q^{\prime} \alpha^{\prime}}} \\
& \left|\hat{\mu}_{k, j}\left(\xi_{1}, \xi_{2}\right)-\hat{\mu}_{k, j}\left(\xi_{1}, 0\right)-\hat{\mu}_{k, j}\left(0, \xi_{2}\right)+\hat{\mu}_{k, j}(0,0)\right| \leq C A^{\prime}\left|\rho^{k} \xi_{1}\right|^{\frac{1}{2 q^{\prime} \alpha^{\prime}}}\left|\rho^{j} \xi_{2}\right|^{\frac{1}{2 q^{\prime} \alpha^{\prime}}}
\end{aligned}
$$

where $C$ is independent of $q, \Omega, h, \alpha$. Choose positive real value functions $\phi_{j} \in C_{0}^{\infty}(\mathbb{R})(j=1,2)$ satisfying $\operatorname{supp}\left(\varphi_{j}\right) \subset\{|r|<1\}$ and $\varphi_{j}=1$, when $|r|<\frac{1}{2}$. Define

$$
\begin{aligned}
& \left(\Phi_{k}^{1}\right)^{\Lambda}\left(\xi_{1}\right)=\phi_{1}\left(\left|\rho^{k} \xi_{1}\right|\right), \\
& \left(\Phi_{j}^{2}\right)^{\Lambda}\left(\xi_{2}\right)=\phi_{2}\left(\left|\rho^{j} \xi_{2}\right|\right),
\end{aligned}
$$

and measures

$$
\begin{aligned}
& \hat{\tau}_{k, j}(\xi)=\hat{\mu}_{k, j}(\xi)-\left(\Phi_{k}^{1}\right)^{\Lambda}\left(\xi_{1}\right) \hat{\mu}_{k, j}\left(0, \xi_{2}\right) \\
& -\left(\Phi_{j}^{2}\right)^{\Lambda}\left(\xi_{2}\right) \hat{\mu}_{k, j}\left(\xi_{1}, 0\right)+\left(\Phi_{k}^{1}\right)^{\Lambda}\left(\xi_{1}\right)\left(\Phi_{j}^{2}\right)^{\Lambda}\left(\xi_{2}\right) \hat{\mu}_{k, j}(0,0) .
\end{aligned}
$$

So by the definition of $\tau_{k, j}$ and estimates (2.18)-(2.21), it is easy to check that $\tau_{k, j}$ satisfies the same estimates as $\sigma_{k, j}$, i.e.,

$$
\left|\hat{\tau}_{k, j}\left(\xi_{1}, \xi_{2}\right)\right| \leq C A^{\prime}\left|\rho^{k} \xi_{1}\right|^{ \pm \frac{1}{2 q^{\prime} \alpha^{\prime}}}\left|\rho^{j} \xi_{2}\right|^{ \pm \frac{1}{2 q^{\prime} \alpha^{\prime}}},
$$

where $C$ is independent of $q, \alpha$ and $\Omega, h$. Also we have

$$
\begin{aligned}
\mu^{*} f\left(x_{1}, x_{2}\right) \leq \sup _{k, j}\left(\Phi_{k}^{1} \otimes \mu_{k, j}^{(1)}\right) * f\left(x_{1}, x_{2}\right) & +\sup _{k, j}\left(\mu_{k, j}^{(2)} \otimes \Phi_{j}^{2}\right) * f\left(x_{1}, x_{2}\right) \\
& +\sup _{k, j}\left(\mu_{k, j}^{(1,2)} \otimes \Phi_{k}^{1} \otimes \Phi_{j}^{2}\right) * f\left(x_{1}, x_{2}\right)+g(f)\left(x_{1}, x_{2}\right),
\end{aligned}
$$

where

$$
g(f)\left(x_{1}, x_{2}\right)=\left(\sum_{k, j}\left|\tau_{k, j} * f\left(x_{1}, x_{2}\right)\right|^{2}\right)^{\frac{1}{2}}
$$

and $\mu_{k, j}^{(1)}, \mu_{k, j}^{(2)}$ and $\mu_{k, j}^{(1,2)}$ defined as follows:

$$
\hat{\mu}_{k, j}^{(1)}\left(\xi_{2}\right)=\hat{\mu}_{k, j}\left(0, \xi_{2}\right), \hat{\mu}_{k, j}^{(2)}\left(\xi_{1}\right)=\hat{\mu}_{k, j}\left(\xi_{1}, 0\right), \hat{\mu}_{k, j}^{(1,2)}\left(\xi_{1}, \xi_{2}\right)=\hat{\mu}_{k, j}(0,0) .
$$

Then, we have

$$
\begin{gathered}
\sup _{k, j}\left(\Phi_{j}^{1} \otimes \mu_{k, j}^{(1)} * f\left(x_{1}, x_{2}\right) \leq C M_{1} M^{(1)} f\left(x_{1}, x_{2}\right)\right. \\
\sup _{k, j}\left(\mu_{k, j}^{(2)} \otimes \Phi_{j}^{2}\right) * f\left(x_{1}, x_{2}\right) \leq C M_{2} M^{(2)} f\left(x_{1}, x_{2}\right) \\
\sup _{k, j}\left(\mu_{k, j}^{(1,2)} \otimes \Phi_{k}^{1} \otimes \Phi_{j}^{2}\right) * f\left(x_{1}, x_{2}\right) \leq C M_{1} M_{2} f\left(x_{1}, x_{2}\right) \hat{\mu}_{k, j}(0,0)
\end{gathered}
$$

where $M_{i}$ is the Hardy-Littlewood maximal function acting on the $x_{i}$-variable and $M$ ${ }^{(i)}$ is the partial maximal function, defined as the following 


$$
M^{(i)} g_{i}=\sup _{k, j}\left|\mu_{k, j}^{(i)} * g_{i}\right|, i=1,2
$$

Since

$$
\begin{aligned}
M^{(1)} g_{1}\left(x_{2}\right) & \leq \sup _{k, j} \int_{\rho^{k}}^{\rho^{k+1}} \int_{\rho^{j}}^{\rho^{j+1}} \int_{S^{n-1} \times S^{m-1}}|\Omega(u, v)||h(r, s)|\left|g\left(x_{2}-s v\right)\right| \mathrm{d} \sigma(u) \mathrm{d} \sigma(v) \frac{\mathrm{d} r \mathrm{~d} s}{r s} \\
& \leq \sup _{k, j} C \log \rho \int_{\rho^{j}} \int_{S^{m-1}} \int_{S^{n-1}}|\Omega(u, v)| \mathrm{d} \sigma(u) \int_{2^{k}}^{2^{k+1}}|h(r, s)| \frac{\mathrm{d} r}{r}\left|g\left(x_{2}-s v\right)\right| \mathrm{d} \sigma(v) \frac{\mathrm{d} s}{s} .
\end{aligned}
$$

We let $\bar{h}(s)=\sup _{k} \int_{2^{k}}^{2^{k+1}}|h(r, s)| \frac{\mathrm{d} r}{r}$ and $\bar{\Omega}(v)=\int_{S^{n-1}}|\Omega(u, v)| \mathrm{d} \sigma(u)$. Since $h \in \widetilde{\Delta}_{\alpha}$ and $\Omega \in L^{q}\left(S^{n-1} \times S^{m-1}\right)$, then $\bar{h} \in \Delta_{\alpha}\left(\mathbb{R}^{+}\right)$and $\bar{\Omega} \in L^{q}\left(S^{m-1}\right)$. By Lemma 1 of [8], the oneparameter case, we have for $p>1+\theta$,

$$
\begin{aligned}
\left\|M^{(1)} g_{1}\right\|_{L^{p}\left(\mathbb{R}^{m}\right)} & \leq \operatorname{Clog}^{2} \rho\|\Omega\|_{L^{q}\left(S^{n-1} \times S^{m-1}\right)}\|h\|_{\tilde{\Delta}_{\alpha}}\left(1-2^{-\frac{\theta}{2}}\right)^{-\frac{2}{p}}\left\|g_{1}\right\|_{L^{p}\left(\mathbb{R}^{m}\right)} \\
& \leq C A^{\prime} B^{\frac{2}{p}}\left\|g_{1}\right\|_{L^{p}\left(\mathbb{R}^{m}\right)}
\end{aligned}
$$

and the same way we have

$$
\begin{aligned}
& \left\|M^{(2)} g_{2}\right\|_{L^{p}\left(\mathbb{R}^{n}\right)} \leq \operatorname{Clog}^{2} \rho\|\Omega\|_{L^{q}\left(S^{n-1} \times S^{m-1}\right)}\|h\|_{\tilde{\Delta}_{\alpha}}\left(1-2^{-\frac{\theta}{p}}\right)^{-\frac{2}{p}}\left\|g_{2}\right\|_{L^{p}\left(\mathbb{R}^{n}\right)} \\
& \leq C A^{\prime} B^{\frac{2}{p}}\left\|g_{2}\right\|_{L^{p}\left(\mathbb{R}^{n}\right)} \text {. }
\end{aligned}
$$

On the other hand, it is easy to check,

$$
\sup _{k, j} \mu_{k, j}^{(1,2)} * f\left(x_{1}, x_{2}\right) \leq C \log ^{2} \rho\|\Omega\|_{L^{q}\left(S^{n-1} \times S^{m-1}\right)}\|h\|_{\Delta_{\alpha}}\left|f\left(x_{1}, x_{2}\right)\right| .
$$

So with (2.28)-(2.30) and (2.25), we concluded that for $p \in(1+\theta, 2]$,

$$
\begin{gathered}
\left\|\sup _{k . j}\left(\Phi_{j}^{1} \otimes \mu_{k . j}^{(1)}\right) * f\right\|_{L^{p}\left(\mathbb{R}^{n} \times \mathbb{R}^{m}\right)} \leq C A^{\prime} B^{\frac{2}{p}}\|f\|_{L^{p}\left(\mathbb{R}^{n} \times \mathbb{R}^{m}\right)} \\
\left\|\sup _{k, j}\left(\mu_{k, j}^{(2)} \otimes \Phi_{j}^{2}\right) * f\right\|_{L^{p}\left(\mathbb{R}^{n} \times \mathbb{R}^{m}\right)} \leq C A^{\prime} B^{\frac{2}{p}}\|f\|_{L^{p}\left(R^{n} \times R^{m}\right),} \\
\left\|\sup _{k, j}\left(\mu_{k, j}^{(1,2)} \otimes \Phi_{k}^{1} \otimes \Phi_{j}^{2}\right) * f\right\|_{L^{p}\left(\mathbb{R}^{n} \times \mathbb{R}^{m}\right)} \leq C A^{\prime} B^{\frac{2}{p}}\|f\|_{L^{p}\left(\mathbb{R}^{n} \times \mathbb{R}^{m}\right) .}
\end{gathered}
$$

To prove Lemma 2.1, it suffices to prove $\|g(f)\|_{L^{p}\left(\mathbb{R}^{n} \times \mathbb{R}^{m}\right)} \leq C A B^{\frac{2}{p}}\|f\|_{L^{p}\left(\mathbb{R}^{n} \times \mathbb{R}^{m}\right)}$ for $p$ $\in(1+\theta, 2]$. By a well-known property of Rademacher's function, this follows from

$$
\|U(f)\|_{L^{p}\left(\mathbb{R}^{n} \times \mathbb{R}^{m}\right)} \leq C A^{\prime} B^{\frac{2}{p}}\|f\|_{L^{p}\left(\mathbb{R}^{n} \times \mathbb{R}^{m}\right)}
$$

for $p \in(1+\theta, 2]$, where $U(f)=\sum_{k, j} \varepsilon_{k, j} \tau_{k, j} * f$ with $\varepsilon_{k, j}=1$ or -1 , and the constant $C$ is independent of $\varepsilon_{k, j}$. The estimate (2.32) is a consequence of the following lemma:

Lemma 2.4. We define a sequence $\left\{p_{j}\right\}_{j=1}^{\infty}$ by $p_{1}=2$ and $\frac{1}{p_{j+1}}=\frac{1}{2}+\frac{1-\theta}{2 p_{j}}$ for $j \geq 1$. (We note that $\frac{1}{p_{j}}=\frac{1-a^{j}}{1+\theta}$, where $a=\frac{1-\theta}{2}$, so $\left\{p_{j}\right\}$ is decreasing and converges to $1+\theta$.) Then, for $j \geq 1$ we have 


$$
\|U(f)\|_{L^{p_{j}}\left(\mathbb{R}^{n} \times \mathbb{R}^{m}\right)} \leq C A^{\prime} B^{2 / p_{j}}\|f\|_{L^{p_{j}}\left(\mathbb{R}^{n} \times \mathbb{R}^{m}\right)} .
$$

Proof. Let

$$
U_{k, m}(f)=\sum_{k, j} \varepsilon_{k, j} \tau_{k, j} *\left(\not_{k+l}^{1} \otimes \not p_{j+m}^{2}\right) *\left(\not_{k+l}^{1} \otimes \not p_{j+m}^{2}\right) * f
$$

By Plancherel's theorem and the estimates (2.23), the same way as in (2.11), we have that

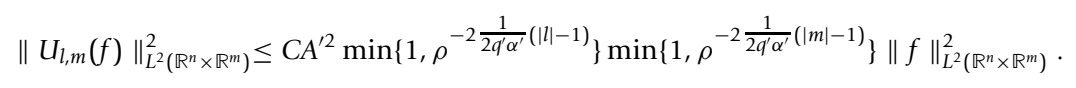

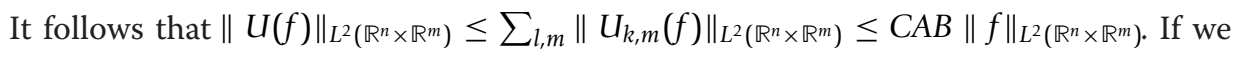
denote by $A(s)$ the claim of Lemma 2.4 for $j=s$, this proves $A(1)$.

Now, we derive $A(s+1)$ from $A(s)$ assuming that $A(s)$ holds, which will complete the proof of Lemma 2.4 by induction. By (2.22) and (2.24), we have that

$$
\begin{aligned}
\tau^{*}(f)(x) & \leq \mu^{*}(|f|)(x)+\sup _{k, j}\left(\Phi_{k}^{1} \otimes \mu_{k, j}^{(1)}\right) * f(x)+\sup _{k, j}\left(\mu_{k, j}^{(2)} \otimes \Phi_{j}^{2}\right) * f(x) \\
& +\sup _{k, j}\left(\mu_{k, j}^{(1,2)} \otimes \Phi_{k}^{1} \otimes \Phi_{j}^{2}\right) * f(x) \\
& \leq g(f)(x)+2\left(\sup _{k, j}\left(\Phi_{k}^{1} \otimes \mu_{k, j}^{(1)}\right) * f(x)+\sup _{k, j}\left(\mu_{k, j}^{(2)} \otimes \Phi_{j}^{2}\right) * f(x)\right. \\
& \left.+\sup _{k, j}\left(\mu_{k, j}^{(1,2)} \otimes \Phi_{k}^{1} \otimes \Phi_{j}^{2}\right) * f(x)\right)
\end{aligned}
$$

Note that $A_{s}$ means that $\|g(f)\|_{p_{s}} \leq C A^{\prime} B^{\frac{2}{p_{s}}}\|f\|_{p_{s}}$. By (2.35) and (2.31) we have

$$
\begin{aligned}
\left\|\tau^{*}(f)\right\|_{L^{s}\left(\mathbb{R}^{n} \times \mathbb{R}^{m}\right)} & \leq\|g(f)\|_{L_{s s}\left(\mathbb{R}^{n} \times \mathbb{R}^{m}\right)}+2\left(\left\|\sup _{k, j}\left(\Phi_{j}^{1} \otimes \mu_{k, j}^{(1)}\right) * f\right\|_{L_{s s}\left(\mathbb{R}^{n} \times \mathbb{R}^{m}\right)}\right. \\
& +\left\|\sup _{k, j}\left(\mu_{k, j}^{(2)} \otimes \Phi_{j}^{2}\right) * f\right\|_{L^{s}\left(\mathbb{R}^{n} \times \mathbb{R}^{m}\right)}+\left\|\sup _{k, j}\left(\mu_{k, j}^{(1,2)} \otimes \Phi_{k}^{1} \otimes \Phi_{j}^{2}\right) * f\right\|_{L_{s}\left(\mathbb{R}^{n} \times \mathbb{R}^{m}\right)} \\
& \leq C A^{\prime} B^{\frac{2}{p_{s}}}\|f\|_{L_{s}\left(\mathbb{R}^{n} \times \mathbb{R}^{m}\right) .}
\end{aligned}
$$

By (2.36) and (2.34), we can now apply the arguments used in the proof of (2.17) to get $A(s+1)$. This completes the proof of Lemma 2.4 .

Now, we prove the inequality (2.32) for $p \in(1+\theta, 2]$. Let $\left\{p_{j}\right\}_{j=1}^{\infty}$ be as in Lemma 2.4. Then, we have $p_{N+1} \leq p \leq p_{N}$ for some $N$. Thus, interpolation between the estimates of Lemma 2.4 for $j=N$ and $j=N+1$, we have (2.36). This completes the proof of Lemma 2.1.

\section{Proofs of Theorem 1.1 and Corollary $\mathbf{1 . 1}$}

Proof of Theorem 1.1: We first need to establish a suitable decomposition for $\Omega$ defined on $S^{n-1} \times S^{m-1}$. The main technique is mainly based on Chen [18]. Define a sequence of sets $\left\{F_{k}\right\}(k \in \mathbb{N})$ on $S^{n-1} \times S^{m-1}$ as:

$$
F_{\kappa}=\left\{(\theta, w) \in S^{n-1} \times S^{m-1}: 2^{\kappa-1} \leq|\Omega(\theta, w)|<2^{\kappa}\right\} \text { for } \kappa=2,3, \ldots
$$

and

$$
F_{1}=\left\{(\theta, w) \in S^{n-1} \times S^{m-1}:|\Omega(\theta, w)|<2\right\} \text { for } \kappa=1 .
$$


We define

$$
\begin{aligned}
& \Omega_{\kappa}(\theta, w)=\Omega \chi_{F_{\kappa}}(\theta, w)-\frac{1}{\sigma\left(S^{n-1}\right)} \int_{S^{n-1}} \Omega \chi_{F_{k}}(u, w) \mathrm{d} \sigma(u) \\
& \quad-\frac{1}{\sigma\left(S^{m-1}\right)} \int_{S^{m-1}} \Omega \chi_{F_{k}}(\theta, v) \mathrm{d} \sigma(v) \\
& \quad+\frac{1}{\sigma\left(S^{n-1}\right) \sigma\left(S^{m-1}\right)} \int_{S^{n-1} \times S^{m-1}} \Omega \chi_{F_{k}}(u, v) \mathrm{d} \sigma(u) \sigma(v) .
\end{aligned}
$$

Then, it is easy to check that

$$
\Omega(\theta, w)=\sum_{\kappa=1}^{\infty} \Omega_{\kappa}(\theta, w)
$$

and all $\Omega_{\kappa}$ satisfies the condition (1.3), i.e.,

$$
\int_{S^{n-1}} \Omega_{\kappa}(\theta, w) \mathrm{d} \sigma(\theta)=\int_{S^{m-1}} \Omega_{\kappa}(\theta, w) \mathrm{d} \sigma(w)=0
$$

Furthermore, if we set $e_{\kappa}=\sigma\left(F_{\kappa}\right)=\int_{F_{\kappa}} \mathrm{d} \sigma(u) d \sigma(v)$, then for $r \in(1, \infty)$, we have

$$
\left\|\Omega_{\kappa}\right\|_{L^{r}\left(S^{n-1} \times S^{m-1}\right)} \leq C 2^{\kappa} e_{\kappa}^{\frac{1}{r}} \text { for } \kappa \in \mathbb{N} .
$$

Now, fix $h \in \tilde{\Delta}_{\alpha}(\alpha \in(1,2]), p \in(1, \infty)$ and a function $f$ with $\|f\|_{L^{p}\left(\mathbb{R}^{n+m}\right)} \leq 1$, we denote

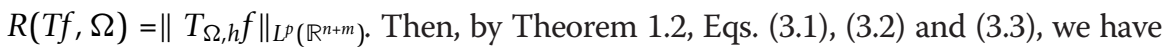

$$
\begin{aligned}
& R(T f, \Omega) \leq \sum_{\kappa=1}^{\infty} R\left(T f, \Omega_{\kappa}\right) \leq C \frac{1}{(\alpha-1)^{2}}\|h\|_{\tilde{\Delta}_{a}} \sum_{\kappa=1}^{\infty} \kappa^{2}\left\|\Omega_{\kappa}\right\|_{L^{1+} \frac{1}{\kappa}\left(S^{n-1} \times S^{n-1}\right)} \\
& \leq C \frac{1}{(\alpha-1)^{2}}\|h\|_{\tilde{\Delta}_{\alpha}} \sum_{k=1}^{\infty} \kappa^{2} 2^{\kappa} e_{\kappa}^{\frac{k}{k+1}} \\
& \leq C \frac{1}{(\alpha-1)^{2}}\|h\|_{\tilde{\Delta}_{\alpha}}\left(\sum_{e_{\kappa}<3-\kappa}+\sum_{e_{k} \geq 3^{-k}}\right) \kappa^{2} 2^{k} e_{\kappa}^{\frac{k}{K+1}} \\
& \leq C \frac{1}{(\alpha-1)^{2}}\|h\|_{\tilde{\Delta}_{\alpha}}\left(\sum_{\kappa \geq 1} \kappa^{2} 2^{\kappa} 3^{-\frac{k^{2}}{\kappa+1}}+\sum_{\kappa \geq 1} \kappa^{2} 2^{\kappa} e_{\kappa} \frac{\kappa}{\kappa+1}\right) \\
& \leq C \frac{1}{(\alpha-1)^{2}}\|h\|_{\tilde{\Delta}_{\alpha}}\left(1+\int_{S^{n-1} \times S^{m-1}}|\Omega(\theta, \omega)| \log ^{2}(2+|\Omega(\theta, \omega)|) \mathrm{d} \sigma(\theta) \mathrm{d} \sigma(\omega)\right) .
\end{aligned}
$$

For $p=2$ and a function $f$ with $\|f\|_{L^{2}\left(\mathbb{R}^{n+m}\right)} \leq 1$. Denote $O(h)=\left\|T_{h} f\right\|_{L^{2}\left(\mathbb{R}^{n} \times \mathbb{R}^{m}\right) \text {. Sup- }}$ pose that $h \in \Delta_{\alpha}(\alpha \in(1,2])$ and $\Omega \in L(\log L)^{2}\left(S^{n-1} \times S^{m-1}\right)$, with the same estimate as in (3.4), we have that

$$
O(h) \leq C \frac{1}{(\alpha-1)^{2}}\|h\|_{\Delta_{\alpha}\left(\mathbb{R}^{+} \times \mathbb{R}^{+}\right)}
$$

Put $E_{1}=\left\{(r, s) \in \mathbb{R}^{+} \times \mathbb{R}^{+}:|h(r, s)| \leq 2\right\}$ and $E_{m}=\left\{(r, s) \in \mathbb{R}^{+} \times \mathbb{R}^{+}: 2^{m-1}<|h(r, s)|\right.$ $\left.\leq 2^{m}\right\}$ for $m=2,3, \ldots$. Then, by (3.5), we have

$$
O\left(h_{\chi E_{m}}\right)=\left\|T_{h_{\chi E_{m}}}\right\|_{L^{2}\left(\mathbb{R}^{n} \times \mathbb{R}^{m}\right)} \leq \frac{C}{(\alpha-1)^{2}}\left\|h_{\chi E_{m}}\right\|_{\Delta_{\alpha}\left(\mathbb{R}^{+} \times \mathbb{R}^{+}\right)} .
$$

We follow the extrapolation argument of Zygmund [7]. First we note that

$$
\left\|h_{\chi E_{m}}\right\|_{{ }_{1+\frac{1}{m}}}\left(\mathbb{R}^{+} \times \mathbb{R}^{+}\right) \leq 2^{m} D_{m}^{\frac{m}{m+1}}(h)
$$


for $m \geq 1$, where $D_{m}(h)$ be as in the definition of $N_{\alpha}$ in Section 1. By (3.5) and (3.6), we have

$$
\begin{aligned}
O(h) & \leq \sum_{m \geq 1} O\left(h \chi_{E_{m}}\right) \\
& \leq C \sum_{m \geq 1} m^{2}\left\|h \chi_{E_{m}}\right\|_{\Delta}{ }_{1+\frac{1}{m}}\left(\mathbb{R}^{+} \times \mathbb{R}^{+}\right) \\
& \leq C \sum_{m \geq 1} m^{2} 2^{m} D_{m}^{\frac{m}{m+1}}(h) \\
& =C\left(\underset{D_{m}(h)<3^{-m}}{\Sigma}+\underset{D_{m}(h) \geq 3^{-m}}{\Sigma}\right) m^{2} 2^{m} D_{m}^{\frac{m}{m+1}}(h) \\
& \leq C\left(1+\|h\|_{N_{2}\left(\mathbb{R}^{+} \times \mathbb{R}^{+}\right)}\right)
\end{aligned}
$$

This ends the proof of Theorem 1.1.

Proof of Corollary 1.1: Since $h$ can be written as separate case $h_{1}(r) \cdot h_{2}(s)$, we deal (1) and (2) by the same procession. We only need to prove part (1) of the corollary. Suppose that $\Omega \in L(\log L)^{2}\left(S^{n-1} \times S^{m-1}\right)$ and $h_{1}, h_{2} \in \Delta_{\alpha}\left(\mathbb{R}^{+}\right)$for $\alpha \in(1,2]$, then $h=h_{1} h_{2} \in \tilde{\Delta}_{\alpha}$. By part (2) of Theorem 1.1, for $p \in(1, \infty)$ we have

$$
\begin{aligned}
\|T f\|_{L^{p}\left(\mathbb{R}^{n} \times \mathbb{R}^{m}\right)} & \leq C \frac{1}{(\alpha-1)^{2}}\|h\|_{\tilde{\Delta}_{\alpha}}\|f\|_{L^{p}\left(\mathbb{R}^{n} \times \mathbb{R}^{m}\right)} \\
& \leq C \frac{1}{(\alpha-1)^{2}}\left\|h_{1}\right\|_{\Delta_{\alpha}\left(\mathbb{R}^{+}\right)}\left\|h_{2}\right\|_{\Delta_{\alpha}\left(\mathbb{R}^{+}\right)}\|f\|_{L^{p}\left(\mathbb{R}^{n} \times \mathbb{R}^{m}\right)}
\end{aligned}
$$

Suppose that $\|\Omega\|_{L(\log L)^{2}\left(S^{n-1} \times S^{m-1}\right)} \leq 1,\|f\|_{L^{p}\left(\mathbb{R}^{n} \times \mathbb{R}^{m}\right)} \leq 1$ and $h_{1} \in \Delta_{\alpha}\left(\mathbb{R}^{+}\right)$with $\left\|h_{1}\right\|_{\Delta_{\alpha}\left(\mathbb{R}^{+}\right)} \leq 1$. We define $U\left(h_{2}\right)=\left\|T_{h_{2}} f\right\|_{L^{p}\left(\mathbb{R}^{n} \times \mathbb{R}^{m}\right)}$. Set $E_{1}=\left\{r \in \mathbb{R}^{+}:\left|h_{2}(r)\right| \leq 2\right\}$ and $E_{m}=\left\{r \in \mathbb{R}^{+}: 2^{m-1}<\left|h_{2}(r)\right| \leq 2^{m}\right\}$ for $m \geq 2$. Then, by (3.7), there exists a constant $C$, which is independent of $\alpha$ such that

$$
U\left(h_{2} \chi_{E_{m}}\right) \leq \frac{C}{(\alpha-1)^{2}}\left\|h_{2} \chi_{E_{m}}\right\|_{\Delta_{\alpha}\left(\mathbb{R}^{+}\right)}
$$

for $\alpha \in(1,2]$. We note

$$
\left\|h_{2} \chi_{E_{m}}\right\|_{\Delta}{ }_{1+\frac{1}{m}}\left(\mathbb{R}^{+}\right) \leq 2^{m} d_{m}^{\frac{m}{m+1}}\left(h_{2}\right)
$$

for $m \geq 1$, where $d_{m}(h)$ is as in Section 1. By (3.8) and (3.9), we have

$$
\begin{aligned}
U(h) & \leq \sum_{m \geq 1} O\left(h_{2} \chi_{E_{m}}\right) \\
& \leq C \sum_{m \geq 1} m^{2}\left\|h_{2} \chi_{E_{m}}\right\|_{\Delta}{ }_{1+\frac{1}{m}}\left(\mathbb{R}^{+}\right) \\
& \leq C \sum_{m \geq 1} m^{2} 2^{m} d_{m}^{\frac{m}{m+1}}\left(h_{2}\right) \\
& =C\left(\sum_{D_{m}\left(h_{1}\right)<3^{-m}}+\underset{d_{m}\left(h_{2}\right) \geq 3^{-m}}{\sum^{-m}}\right) m^{2} 2^{m} d_{m}^{\frac{m}{m+1}}\left(h_{2}\right) \\
& \leq C\left(1+N_{2}\left(h_{2}\right)\right),
\end{aligned}
$$

which finishes the proof of the corollary. 


\section{Acknowledgements}

This work originated from the author's dissertation at Beijing normal University under the inspiring guidance of Professor Ding Y. The author would like to express their deep gratitude to the referee for his/her valuable comments and suggestions. The work was partially supported by NSF of China (Grant: 10701010), University NSR Project of Anhui Province (Grant: KJ2011A138) and Scientific Research Foundation of Anhui normal university.

\section{Competing interests}

The authors declare that they have no competing interests.

Received: 9 July 2011 Accepted: 21 November 2011 Published: 21 November 2011

\section{References}

1. Calderón, AP, Zygmund, A: On singular integrals. Amer J Math. 78, 289-309 (1956). doi:10.2307/2372517

2. Fefferman, R: A note on singular integrals. Proc Am Math Soc. 74, 266-270 (1979). doi:10.1090/50002-9939-19790524298-3

3. Namazi, J: A singular integral. Proc Am Math Soc. 96, 421-424 (1986). doi:10.1090/50002-9939-1986-0822432-2

4. Duoandikoetxea, J, Rubio de Francia, JL: Maximal and singular integral operators via fourier transform estimates. Invent Math. 84, 541-561 (1986). doi:10.1007/BF01388746

5. Al-Salman, A, Pan, Y: Singular integrals with rough kernels in $L \log L\left(S^{n-1}\right)$. J Lond Math Soc 66, 153-174 (2002). (2), doi:10.1112/S0024610702003241

6. Yano, S: An extrapolation theorem. J Math Soc Jpn. 3, 296-305 (1951). doi:10.2969/jmsj/00320296

7. Zygmund, A: Trigonometric series. Cambridge University Press, London, Secondl, II (1968) reprinted with corrections and some additions.

8. Sato, S: Estimates for singular integrals and extrapolation. Stud Math. 193, 219-233 (2009)

9. Duoandikoetxea, J: Weighted norm inequalities for homogeneous singular integrals. Trans Amer Math Soc. 336, 869-880 (1993). doi:10.2307/2154381

10. Watson, DK: Weighted estimates for singular integrals via Fourier transform estimates. Duke Math J. 60, 389-399 (1990). doi:10.1215/50012-7094-90-06015-6

11. Stein, EM: Singular integrals and differentiability properties of functions. Princeton University Press, Princeton, NJ (1970)

12. Stein, EM: Harmonic analysis: real-variable methods, orthogonality, and oscillatory integrals. Princeton University Press, Princeton, NJ (1993)

13. Duoandikoetxea, J: Fourier analysis, American Mathematical Society, Providence, RI(2001). Translated and revised from the 1995 Spanish original by David Cruz-Uribe

14. Fefferman, R, Stein, EM: Singular integrals on product spaces. Adv Math. 45, 117-143 (1982). doi:10.1016/50001-8708(82) $80001-7$

15. Duoandikoetxea, J: Multiple singular integrals and maximal functions along hypersurfaces. Ann Inst Fourier. 36, 185-206 (1986). doi:10.5802/aif.1073

16. Fan, D, Guo, K, Pan, Y: Singular integrals with rough kernels on product spaces. Hokkaido Math J. 28, 435-460 (1999)

17. Jiang, YS, Lu, SZ: A class of singular integral operators with rough kernel on product domains. Hokkaido Math J. 24, 1-7 (1995)

18. Chen, J: $L^{p}$ boundedness of singular integrals on product domains. Sci China Ser A. 44, 681-689 (2001)

19. Al-Salman, A, Al-Qassem, H, Pan, Y: Singular integrals on product domains. Indiana Univ Math J. 55, 369-387 (2006). doi:10.1512/iumj.2006.55.2626

20. Al-Qassem, H, Pan, Y: $L^{P}$ boundedness for singular integrals with rough kernels on product domains. Hokkaido Math J. 31, 555-613 (2002)

doi:10.1186/1029-242X-2011-115

Cite this article as: Qu: Estimates for the multiple singular integrals via extrapolation. Journal of Inequalities and Applications 2011 2011:115.

\section{Submit your manuscript to a SpringerOpen ${ }^{\circ}$ journal and benefit from:}

Convenient online submission

- Rigorous peer review

- Immediate publication on acceptance

- Open access: articles freely available online

- High visibility within the field

- Retaining the copyright to your article

Submit your next manuscript at $\boldsymbol{s p r i n g e r o p e n . c o m ~}$ 